\title{
Bringing Again Noble Metal Nanoparticles to the Forefront of Cancer Therapy
}

\author{
Ylea Vlamidis and Valerio Voliani* \\ Center for Nanotechnology Innovation, Istituto Italiano di Tecnologia, Pisa, Italy
}

Nanomaterials have attracted increasing interest for their potentiality to revolutionize the diagnosis and treatment of many diseases, especially neoplasms. Interestingly, there is a huge imbalance between the number of proposed nanoplatforms and the few ones approved for clinical applications. This disequilibrium affects in particular noble metal nanoparticles (NPs), that present no-approved platform and very few candidates in clinical trials because of the issue of persistence. In this perspective, we discuss if nanomedicine is generally keeping its promises with a focus on the approach that could fill the gap between NPs and oncology in the next future: the ultrasmall-in-nano.

OPEN ACCESS

Edited by:

Silke Krol,

Istituto Tumori Giovanni Paolo II

(IRCCS), Italy

Reviewed by:

Valeria Chiono,

Politecnico di Torino, Italy

Alfredo Ambrosone,

Università degli Studi di Salerno, Italy

*Correspondence:

Valerio Voliani

valerio.voliani@it.it

Specialty section:

This article was submitted to

Nanobiotechnology,

a section of the journal

Frontiers in Bioengineering and

Biotechnology

Received: 04 July 2018 Accepted: 19 September 2018 Published: 08 October 2018

Citation:

Vlamidis Y and Voliani V (2018)

Bringing Again Noble Metal

Nanoparticles to the Forefront of

Cancer Therapy.

Front. Bioeng. Biotechnol. 6:143.

doi: 10.3389/fbioe.2018.00143
Keywords: persistence, oncology, nanoparticles, theranostic agent, clinical translation, nanomedicine, nanobiotechnology

\section{THE LACK OF TRANSLATION OF NOBLE METAL NANOPARTICLES}

Nanoplatforms, due to their unique size/shape-influenced chemical, physical, and physiological features, are widespread studied for applications in many fields of academic research. In particular, they should have pushed forward the paradigms in key-sectors such as energy harvesting, environmental remediation, and medicine. About the latter, researchers have profused most of their efforts toward the development of novel or enhanced diagnosis and treatments of neoplasms (Lee et al., 2013; Yamada et al., 2015; Yang et al., 2015; Abadeer and Murphy, 2016). After more than 20 years from the approbation of the first nanomedicine, Doxil-doxorubicin-loaded PEGylated nano-liposomes (Barenholz, 2012) -, the question is: has nanomedicine kept its promises in oncology?

At a glance, the answer is "in part." Indeed, after Doxil, regulatory agencies such as FDA and EMA have approved other 50 "nanodrugs," about other 30 products are undergoing clinical trials and some of them could be on the market in the next future (Anselmo and Mitragotri, 2016; Bobo et al., 2016; Caster et al., 2017). Approved nanoplatforms are mainly composed by lipids (such as Myocet, which is commercially available to treat metastatic breast cancer) or proteins (e.g., Abraxane, which is employed to treat different types of cancer), for a US billions market (Ragelle et al., 2017). In particular, it is expected that the global market of nanopharmaceuticals will reach \$412 billions in 2019 (Ragelle et al., 2017); a market in which cancer therapeutic products such as Abraxane and Doxil play a pivotal role. The enhanced action is commonly related to the size and, in some cases, to the ability to make commonly employed drugs more available to the action site(s) (Ragelle et al., 2017). This means that the full potentiality of nanomaterials to revolutionize the clinical practice in oncology is yet not reached (Chen et al., 2017). Remarkably, despite a number of appealing features to design efficient and less invasive cancer treatments (Dreaden et al., 2012; Sun et al., 2014; Yang et al., 2015), noble metal nanoparticles (NPs) are still on the bench-side. 
Such NPs based on gold, silver, or platinum are widespread investigated in medical applications due to their size and shape dependent optoelectronic properties. In particular, Au NPs have been commonly proposed for cancer therapy and diagnostics, Ag NPs displays interesting antibacterial activity, whereas $\mathrm{Pt}$ NPs can be employed as reactive oxygen species scavengers, due to their ability to catalyze biological reactions. The most common potential applications of noble metal NPs in medicine are summarized in Table $\mathbf{1}$.

It is worth to notice that the lack of translation of noble metal NPs to clinics is mainly related to the issue of persistence (Etheridge et al., 2013; Ehlerding et al., 2015; Bobo et al., 2016).

The persistence of NPs in organisms leads to possible interference with common medical diagnoses and can induce severe damages and gene expression alterations (Khlebtsov and Dykman, 2011; Cassano et al., 2018). Regulatory agencies require a complete clearance from the body of all the pharmaceutical and their components in an acceptable timeframe (Zamboni et al., 2012; Eaton et al., 2015; Sainz et al., 2015). Therefore, NPs have to be designed with proper size, shape, charge, and surface chemistry, in order to ensure the optimal conjugation between action and clearance. The excretion is an essential biological process to eliminate materials from organisms, and can be accomplished by renal or hepatic pathways (Liu et al., 2013). The renal pathway relies on glomerular filtration in kidneys, which size threshold is typically $<8 \mathrm{~nm}$, denoting that only materials with ultrasmall hydrodynamic diameters (HD) can be efficiently eliminated through the urinary system (Du et al., 2017; Wang and Liu, 2018). On the other hand, materials with HD $>8 \mathrm{~nm}$ (and generally metal NPs with intriguing behaviors for theranostic applications show $\mathrm{HD}>8 \mathrm{~nm}$ ) are mainly captured by liver and spleen (Tsoi et al., 2016). If captured materials are biodegradable, the building blocks are then excreted by bile and

TABLE 1 | Main medical applications of noble metal NPs in academic studies.

\begin{tabular}{|c|c|c|}
\hline Type of metal NPs & $\begin{array}{l}\text { Biomedical } \\
\text { applications }\end{array}$ & References \\
\hline \multirow[t]{3}{*}{ Au NPs } & Cancer treatment & Dreaden et al., 2012 \\
\hline & Diagnostic/imaging & Abadeer and Murphy, 2016 \\
\hline & $\begin{array}{l}\text { Osteoinductive agent } \\
\text { during dental implant } \\
\text { therapy }\end{array}$ & Jadhav et al., 2018 \\
\hline \multirow[t]{4}{*}{ Pt NPs } & Cancer treatment & Porcel et al., 2010 \\
\hline & Diagnostic/imaging & Liu et al., 2011 \\
\hline & $\begin{array}{l}\text { Scavengers of reactive } \\
\text { oxygen species }\end{array}$ & Moglianetti et al., 2016 \\
\hline & $\begin{array}{l}\text { Improve dentin bond } \\
\text { strength }\end{array}$ & Hoshika et al., 2010 \\
\hline \multirow[t]{5}{*}{ Ag NPs } & Cancer treatment & Ge et al., 2014 \\
\hline & Antibacterial activity & Li et al., 2010 \\
\hline & Antifungal activity & $\begin{array}{l}\text { Gopinath and Velusamy, } \\
2013\end{array}$ \\
\hline & Antiviral activity & Xiang et al., 2011 \\
\hline & $\begin{array}{l}\text { Dental resin filler } \\
\text { composite }\end{array}$ & Abbasi et al., 2014 \\
\hline
\end{tabular}

feces (Sun et al., 2014; Tsoi et al., 2016). Metal NPs are not easily (bio)degradable from organisms, resulting in their accumulation in the reticuloendothelial system (RES), especially in liver and spleen, for long time after the theranostic action (Khlebtsov and Dykman, 2011; Ali et al., 2017). In general, NPs are internalized by cells through endocytic mechanisms and may be exposed to enzymes comprised in lysosomes. Such agents are able to mediate the degradation/corrosion of metal NPs. In particular, the acidic environment of the vesicles can lead to etching of the NPs, causing the release of relatively toxic free metal ions, as for example $\mathrm{Ag}^{+}$and $\mathrm{Au}^{+} /{ }^{3+}$. Such ions can potentially affect cell homeostasis and functions, causing cytotoxicity (Deng and Gao, 2016). Obviously, the long-term etching leads to a decrease of the NPs diameter, allowing their long-term gradual elimination from the body (Sabella et al., 2014). Overall, kidney passive filtration represents the most reliable excretion route for metal NPs in order to minimize potential side effects resulting from long-term metal persistence (Zarschler et al., 2016; Cassano et al., 2018).

As a first attempt to overcome the issue of persistence, many efforts have been done on the reduction of the size of NPs to ultrasmall range ( $<8 \mathrm{~nm}$, ultrasmall nanoparticles, USNPs) in order to enhance their renal clearance efficiency (Zarschler et al., 2016). Due to this smart approach an interesting level of metal excretion was reached (Zarschler et al., 2016; Schmid et al., 2017), but commonly losing some key-features of NPs, among which physical and physiological behaviors (e.g., localized surface plasmon resonances and passive accumulation in targets; Cassano et al., 2018). It is worth to remember here that USNPs can exhibit cytotoxic profile when the stabilizing ligands allow for direct access to the metal surface either for the direct interaction with biomolecules or for catalytic activity of the unshielded metal surface; this topic is carefully discussed elsewhere (Schmid et al., 2017).

A groundbreaking advance to bring again NPs to the forefront of cancer theranostics relies on the ultrasmall-in-nano approach for the design of nanoplatforms able to jointly combine the unique behaviors of plasmonic NPs with the body excretion by the renal pathway (Cassano et al., 2018).

Ultrasmall-in-nano platforms are structures usually composed by aggregates of plasmonic USNPs comprised in (bio)degradable nanocapsules. A significant example in this direction are the nature-inspired passion fruit-like nanoarchitectures (NAs) (Cassano et al., 2015, 2017). NAs are produced by an extreme versatile and robust synthetic protocol (Cassano et al., 2017; Pocovi-Martinez et al., 2018). NAs biodegrade in $<48 \mathrm{~h}$ to polymers, endogenous-glutathione (GSH) coated plasmonic gold USNPs and silicic acid (Cassano et al., 2016; Avigo et al., 2017). The remarkable biocompatibility of NAs and of the relative building blocks was assessed in cultured cells and Zebrafish models (Cassano et al., 2016; d'Amora et al., 2018). Furthermore, some of their potential applications as drug delivery vehicles and imaging contrast agents were already demonstrated, pointing out that all the components of NAs are pivotal (Cassano et al., 2016; Avigo et al., 2017; Armanetti et al., 2018; Mapanao et al., 2018).

Due to the peculiar structure of ultrasmall-in-nano platforms, toxicity issues related to long-term accumulation in organs 
are overcome, unlocking the true potential of noble metal nanotheranostics for cancer treatment and beyond.

Nevertheless, it is interesting to notice that despite the issue of persistence is well-known, there are still an increasing number of publications, also in top tiers Journals, about the design of non-excretable metal nanoplatforms with intriguing features and increasingly complex structure-meaning a number of, for example, imaging and therapeutic applications on the same platform-, but without any real potentiality of translation. At this point the question is: if this kind of NPs cannot be translated to clinics, why is there this trend? Indeed, the main interesting behaviors of metal NPs are now well described together with their potential applications as innovative or synergistic nanoplatforms for theranostics. Moreover, more complex is a nanoplatform and higher will be its intrinsic cost (i.e., market potentiality is strongly reduced; Malinoski, 2014).

The context described together with the lack of an "industrial culture" of many researchers help to explain the huge imbalance between the metal NPs proposed on peer-reviewed journals and the very few candidates in clinical trials (listed in Table 2). Besides basic research, which has to be always encouraged, an ideal approach to hold the promises of nanomedicine in the very next future should be to: (i) develop the most promising leading candidates that overcome significant key-issues (such as toxicity and persistence in organism after the medical action), and (ii) design novel NPs with the aim to overcome a specific issue, in order to focus on translational research.

\section{GENERAL REMARKS}

As pointed out in the previous section, the market of nanoparticles for clinical applications should increase from 15\% of the total pharmaceutical market in 2014 to $22 \%$ in 2019 mainly thanks to the release of nanoplatforms that have increased the efficiency of well-known drugs (Ragelle et al., 2017). On the other hand, the next revolution in oncology has still to come, and it will probably relate to the release of ultrasmall-in-nano noble metal nanoparticles, which will be able to jointly combine innovative, synergistic and non-invasive treatments for a number of neoplasms avoiding the issue of metal persistence in the organism.

Nevertheless, some other topics have to be considered while designing a nanoplatform. For example, it is pivotal to have a deep understanding of the physicochemical properties of the materials, and to develop reasonable, safe, reproducible and scalable synthesis procedures for the production of sterilized products. These subjects are comprehensively discussed elsewhere (Zamboni et al., 2012; Sainz et al., 2015). In order to accelerate the transition of basic nanoscale particles and devices into clinical applications, some organizations have recently emerged, such as the Nanotechnology Characterization Laboratory (NCL) in US and the Nanomedicine Characterization Laboratory (EUNCL) in Europe. These organizations provide guidelines for the development and characterizations of nanotheranostics together with a support to researchers by a free preclinical characterization, pharmacokinetic, efficacy, and toxicity testing of promising nanotech cancer therapeutics and diagnostics by employing a standardized "Assay Cascade." A peculiar assay cascade for nanomaterials is obviously desirable, as the structural complexity of NPs makes the regulation guidelines for small molecules somehow inadequate and insufficient. For example, the features of NPs can be easily altered not only by slight changes in raw materials, but also by small modifications in manufacturing processes, that can result in limited alterations of the structure but in significantly altered biological and biodistribution behaviors. Furthermore, it would also be desirable the development of peculiar diagnostic methods/probes able to follow nanotheranostics effects without interacting nor interfering with the nanoplatforms. Indeed, therapeutic response monitoring should provide feedback that can guide follow-up regimens. Another aspect, which has yet to be fully understood, is related to the responsivity of neoplasms, which can also significantly change between patients. Considering this aspect, a complete preclinical evaluation of the behaviors of nanoplatforms (safety toxicology and absorption/distribution/metabolism/excretion-ADME package) should be obtained in a couple of in vivo models.

A quite underestimated topic related to the employment of metal NPs in clinical practice is the environmental safety assessment. Since 2011, the European Commission is focusing on the risks related to the fate of nanomaterials in the air, water, waste, industrial emissions, to provide more insight for further legislative guidance and risk assessment. Indeed, there is still a considerable lack of data on both the exposure to nanomaterials via the environment and to the exposure of environment to human-made nanomaterials. Consequently,

TABLE 2 | List of the principal nanomedicines based on metal NPs in clinical trials (source: ClinicalTrials.gov).

\begin{tabular}{|c|c|c|c|c|}
\hline Name & Nanomedicine & NPs diameter (nm) & Indication & Status \\
\hline AuroLase & PEG-coated silica-gold nanoshells & $1-3$ & $\begin{array}{l}\text { Primary and/ } \\
\text { or metastatic lung tumors }\end{array}$ & Phase I \\
\hline AGulX & Gadolinium-based NPs & 3 & Brain metastases & Phase I \\
\hline Magnablate & Magnetic iron NPs & Not available & Prostate cancer & Phase I \\
\hline Clariscan & Iron Oxide NPs & $5-7$ & Enhanced MRI Contrast & Phase III \\
\hline NBTXR3 & Hafnium oxide NPs & 50 & $\begin{array}{l}\text { Locally advanced } \\
\text { squamous cell } \\
\text { carcinoma }\end{array}$ & Phase $1 / 11$ \\
\hline- & Silver NPs & $12-15$ & Inflammatory diseases & Phase I \\
\hline
\end{tabular}


no specific provisions for nanomaterials employment have yet been established by EU legislation (Second Regulatory Review on Nanomaterials COM/2012/0572). The EU Commission is currently working on detection and monitoring methods for nanomaterials and their validation to ensure the proper implementation of the norms.

\section{CONCLUSION}

Overall, in order to promote the translation of the new findings to patients, extended collaborations involving scientists from the Health Agencies as well as academic, clinicians, and industry scientists would be fundamental. Besides an extended collaboration, it would be worth to establish a transnational coordination and funding in a flagship framework fashion. Last but not least, researchers should:

\section{REFERENCES}

Abadeer, N. S., and Murphy, C. J. (2016). Recent progress in cancer thermal therapy using gold nanoparticles. J. Phys. Chem. C 120, 4691-4716. doi: 10.1021/acs.jpcc.5b11232

Abbasi, E., Milani, M., Fekri Aval, S., Kouhi, M., Akbarzadeh, A., Tayefi Nasrabadi, H., et al. (2014). Silver nanoparticles: synthesis methods, bio-applications and properties. Crit. Rev. Microbiol. 42, 173-180. doi: 10.3109/1040841X.2014.912200

Ali, M. R. K., Rahman, M. A., Wu, Y., Han, T., Peng, X., Mackey, M. A., et al. (2017). Efficacy, long-term toxicity, and mechanistic studies of gold nanorods photothermal therapy of cancer in xenograft mice. Proc. Natl. Acad. Sci. U.S.A. 114, E3110-E3118. doi: 10.1073/pnas.1619302114

Anselmo, A. C., and Mitragotri, S. (2016). Nanoparticles in the clinic. Bioeng. Transl. Med. 1, 10-29. doi: 10.1002/btm2.10003

Armanetti, P., Pocoví-Martínez, S., Flori, A., Avigo, C., Cassano, D., Menichetti, L., et al. (2018). Dual photoacoustic/ultrasound multi-parametric imaging from passion fruit-like nano-architectures. Nanomed. Nanotechnol. Biol. Med. 14, 1787-1795. doi: 10.1016/j.nano.2018.05.007

Avigo, C., Cassano, D., Kusmic, C., Voliani, V., and Menichetti, L. (2017). Enhanced photoacoustic signal of passion fruit-like nanoarchitectures in a biological environment. J. Phys. Chem. C 121, 6955-6961. doi: 10.1021/acs.jpcc.6b11799

Barenholz, Y. (2012). Doxil $\AA$ - The first FDA-approved nano-drug: lessons learned. J. Control. Release 160, 117-134. doi: 10.1016/j.jconrel.2012.03.020

Bobo, D., Robinson, K. J., Islam, J., Thurecht, K. J., and Corrie, S. R. (2016). Nanoparticle-based medicines: a review of fda-approved materials and clinical trials to date. Pharm. Res. 33, 2373-2387. doi: 10.1007/s11095-016-1958-5

Cassano, D., David, J., Luin, S., and Voliani, V. (2017). Passion fruitlike nano-architectures: a general synthesis route. Sci. Rep. 7:43795. doi: $10.1038 /$ srep43795

Cassano, D., Pocoví-Martínez, S., and Voliani, V. (2018). Ultrasmall-in-nano approach: enabling the translation of metal nanomaterials to clinics. Bioconjug. Chem. 29, 4-16. doi: 10.1021/acs.bioconjchem.7b00664

Cassano, D., Rota Martir, D., Signore, G., Piazza, V., and Voliani, V. (2015). Biodegradable hollow silica nanospheres containing gold nanoparticle arrays. Chem. Commun. 51, 9939-9941. doi: 10.1039/C5CC02771C

Cassano, D., Santi, M., Cappello, V., Luin, S., Signore, G., and Voliani, V. (2016). Biodegradable passion fruit-like nano-architectures as carriers for cisplatin prodrug. Part. Part. Syst. Charact. 33, 818-824. doi: 10.1002/ppsc.201600175

Caster, J. M., Patel, A. N., Zhang, T., and Wang, A. (2017). Investigational nanomedicines in 2016: a review of nanotherapeutics currently undergoing clinical trials. Wiley Interdiscip. Rev. Nanomed. Nanobiotechnol. 9:e1416. doi: 10.1002/wnan.1416

Chen, H., Zhang, W., Zhu, G., Xie, J., and Chen, X. (2017). Rethinking cancer nanotheranostics. Nat. Rev. Mater. 2:17024. doi: 10.1038/natrevmats.2017.24 (i) employ a part of their time to disseminate science and their results to the community and to students in order to increase the science awareness in the society, and (ii) avoid to overemphasize their findings in peer-reviewed manuscripts, as such practice may discourage public confidence in nanomedicine.

\section{AUTHOR CONTRIBUTIONS}

YV and VV have written the manuscript and are familiar with its content.

\section{ACKNOWLEDGMENTS}

VV thank Associazione Italiana per la Ricerca sul Cancro (AIRC) for the individual grant MFAG number 19852.

d'Amora, M., Cassano, D., Pocoví-Martínez, S., Giordani, S., and Voliani, V. (2018). Biodistribution and biocompatibility of passion fruit-like nano-architectures in zebrafish. Nanotoxicology 22, 1-9. doi: 10.1080/17435390.2018.1498551

Deng, J., and Gao, C. (2016). Recent advances in interactions of designed nanoparticles and cells with respect to cellular uptake, intracellular fate, degradation and cytotoxicity. Nanotechnology 27:412002. doi: 10.1088/0957-4484/27/41/412002

Dreaden, E. C., Alkilany, A. M., Huang, X., Murphy, C. J., and El-Sayed, M. A. (2012). The golden age: gold nanoparticles for biomedicine. Chem. Soc. Rev. 41, 2740-2779. doi: 10.1039/C1CS15237H

Du, B., Jiang, X., Das, A., Zhou, Q., Yu, M., Jin, R., et al. (2017). Glomerular barrier behaves as an atomically precise bandpass filter in a subnanometre regime. Nat. Nanotechnol. 12, 1096-1102. doi: 10.1038/nnano. 2017.170

Eaton, M. A. W., Levy, L., and Fontaine, O. M. A. (2015). Delivering nanomedicines to patients: a practical guide. Nanomed. Nanotechnol. Biol. Med. 11, 983-992. doi: 10.1016/j.nano.2015.02.004

Ehlerding, E. B., Chen, F., and Cai, W. (2015). Biodegradable and renal clearable inorganic nanoparticles. Adv. Sci. 3:1500223. doi: 10.1002/advs.201500223

Etheridge, M. L., Campbell, S. A., Erdman, A. G., Haynes, C. L., Wolf, S. M., and McCullough, J. (2013). The big picture on nanomedicine: the state of investigational and approved nanomedicine products. Nanomed. Nanotechnol. Biol. Med. 9, 1-14. doi: 10.1016/j.nano.2012.05.013

Ge, L., Li, Q., Wang, M., Ouyang, J., Li, X., and Xing, M. M. Q. (2014). Nanosilver particles in medical applications: Synthesis, performance, and toxicity. Int. J. Nanomed. 9, 2399-2407. doi: 10.2147/IJN.S55015

Gopinath, V., and Velusamy, P. (2013). Extracellular biosynthesis of silver nanoparticles using Bacillus sp. GP-23 and evaluation of their antifungal activity towards Fusarium oxysporum. Spectrochim. Acta Part A Mol. Biomol. Spectrosc. 106, 170-174. doi: 10.1016/j.saa.2012.12.087

Hoshika, S., Nagano, F., Tanaka, T., Ikeda, T., Wada, T., Asakura, K., et al. (2010). Effect of application time of colloidal platinum nanoparticles on the microtensile bond strength to dentin. Dent. Mater. J. 29, 682-689. doi: 10.4012/dmj.2009-125

Jadhav, K., HR, R., Deshpande, S., Jagwani, S., Dhamecha, D., Jalalpure, S., et al. (2018). Phytosynthesis of gold nanoparticles: characterization, biocompatibility, and evaluation of its osteoinductive potential for application in implant dentistry. Mater. Sci. Eng. C. doi: 10.1016/j.msec.2018. 08.028

Khlebtsov, N., and Dykman, L. (2011). Biodistribution and toxicity of engineered gold nanoparticles: a review of in vitro and in vivo studies. Chem. Soc. Rev. 40, 1647-1671. doi: 10.1039/C0CS00018C

Lee, N., Choi, S. H., and Hyeon, T. (2013). Nano-sized CT contrast agents. Adv. Mater. Weinheim. 25, 2641-2660. doi: 10.1002/adma.2013 00081 
Li, W. R., Xie, X. B., Shi, Q. S., Zeng, H. Y., Ou-Yang, Y. S., and Chen, Y., Ben (2010). Antibacterial activity and mechanism of silver nanoparticles on Escherichia coli. Appl. Microbiol. Biotechnol. 85, 1115-1122. doi: 10.1007/s00253-009-2159-5

Liu, J., Yu, M., Zhou, C., and Zheng, J. (2013). Renal clearable inorganic nanoparticles: a new frontier of bionanotechnology. Mater. Today 16, 477-486. doi: 10.1016/j.mattod.2013.11.003

Liu, Y., Li, D., and Sun, S. (2011). Pt-based composite nanoparticles for magnetic, catalytic, and biomedical applications. J. Mater. Chem. 21, 12579-12587. doi: $10.1039 / \mathrm{c} 1 \mathrm{jm} 11605 \mathrm{c}$

Malinoski, F. J. (2014). The nanomedicines alliance: an industry perspective on nanomedicines. Nanomed. Nanotechnol. Biol. Med. 10, 1819-1820. doi: 10.1016/j.nano.2014.07.003

Mapanao, A. K., Santi, M., Faraci, P., Cappello, V., Cassano, C., and Voliani, V. (2018). Endogenously triggerable ultrasmall-in-nano architectures: targeting assessment on 3D pancreatic carcinoma spheroids. ACS Omega 3, 11796-11801. doi: 10.1021/acsomega.8b01719

Moglianetti, M., De Luca, E., Pedone, D., Marotta, R., Catelani, T., Sartori, B., et al. (2016). Platinum nanozymes recover cellular ROS homeostasis in an oxidative stress-mediated disease model. Nanoscale 8, 3739-3752. doi: $10.1039 / \mathrm{c} 5 \mathrm{nr} 08358 \mathrm{c}$

Pocovi-Martinez, S., Cassano, D., and Voliani, V. (2018). Naked nanoparticles in silica nanocapsules: a versatile family of nanorattle catalysts. ACS Appl. Nano Mater. 1, 1836-1840. doi: 10.1021/acsanm.8b00247

Porcel, E., Liehn, S., Remita, H., Usami, N., Kobayashi, K., Furusawa, Y., et al. (2010). Platinum nanoparticles: a promising material for future cancer therapy? Nanotechnology 21:85103. doi: 10.1088/0957-4484/21/8/085103

Ragelle, H., Danhier, F., Préat, V., Langer, R., and Anderson, D. G. (2017). Nanoparticle-based drug delivery systems: a commercial and regulatory outlook as the field matures. Expert Opin. Drug Deliv. 14, 851-864. doi: 10.1080/17425247.2016.1244187

Sabella, S., Carney, R. P., Brunetti, V., Malvindi, M. A., Al-Juffali, N., Vecchio, G., et al. (2014). A general mechanism for intracellular toxicity of metal-containing nanoparticles. Nanoscale 6, 7052-7061. doi: 10.1039/c4nr01234h

Sainz, V., Conniot, J., Matos, A. I., Peres, C., Zupanoi,o, E., Moura, L., et al. (2015). Regulatory aspects on nanomedicines. Biochem. Biophys. Res. Commun. 468, 504-510. doi: 10.1016/j.bbrc.2015.08.023

Schmid, G., Kreyling, W. G., and Simon, U. (2017). Toxic effects and biodistribution of ultrasmall gold nanoparticles. Arch. Toxicol. 91, 3011-3037. doi: 10.1007/s00204-017-2016-8
Sun, T., Zhang, Y. S., Pang, B., Hyun, D. C., Yang, M., and Xia, Y. (2014). Engineered nanoparticles for drug delivery in cancer therapy. Angew. Chemie Int. Ed. 53, 12320-12364. doi: 10.1002/anie.2014 03036

Tsoi, K. M., Macparland, S. A., Ma, X. Z., Spetzler, V. N., Echeverri, J., Ouyang, B., et al. (2016). Mechanism of hard-nanomaterial clearance by the liver. Nat. Mater. 15, 1212-1221. doi: 10.1038/nmat4718

Wang, J., and Liu, G. (2018). Imaging Nano-Bio interactions in the kidney: toward a better understanding of nanoparticle clearance. Angew. Chem. Int. Ed. 57, 3008-3010. doi: 10.1002/anie.201711705

Xiang, D., Chen, Q., Pang, L., and Zheng, C. (2011). Inhibitory effects of silver nanoparticles on $\mathrm{H} 1 \mathrm{N1}$ influenza A virus in vitro. J. Virol. Methods. 178, 137-142. doi: 10.1016/j.jviromet.2011. 09.003

Yamada, M., Foote, M., and Prow, T. W. (2015). Therapeutic gold, silver, and platinum nanoparticles. Wiley Interdiscip. Rev. Nanomed. Nanobiotechnol. 7, 428-445. doi: 10.1002/wnan.1322

Yang, X., Yang, M., Pang, B., Vara, M., and Xia, Y. (2015). Gold nanomaterials at work in biomedicine. Chem. Rev. 115, 10410-10488. doi: 10.1021/acs.chemrev.5b00193

Zamboni, W. C., Torchilin, V., Patri, A. K., Hrkach, J., Stern, S., Lee, R., et al. (2012). Best practices in cancer nanotechnology: perspective from NCI nanotechnology alliance. Clin. Cancer Res. 18, 3229-3241. doi: 10.1158/1078-0432.CCR-11-2938

Zarschler, K., Rocks, L., Licciardello, N., Boselli, L., Polo, E., Garcia, K. P., et al. (2016). Ultrasmall inorganic nanoparticles: state-of-the-art and perspectives for biomedical applications. Nanomed. Nanotechnol. Biol. Med. 12, 1663-1701. doi: 10.1016/j.nano.2016.02.019

Conflict of Interest Statement: The authors declare that the research was conducted in the absence of any commercial or financial relationships that could be construed as a potential conflict of interest.

Copyright (C) 2018 Vlamidis and Voliani. This is an open-access article distributed under the terms of the Creative Commons Attribution License (CC BY). The use, distribution or reproduction in other forums is permitted, provided the original author(s) and the copyright owner(s) are credited and that the original publication in this journal is cited, in accordance with accepted academic practice. No use, distribution or reproduction is permitted which does not comply with these terms. 